all eligible patients have been targeted, and to exclude a "dropoff" in compliance.

\section{G81(P) SAFETY OF "SINGLE CHECKER" PATIENT GROUP DIRECTIVES FOR SELECTED MEDICATIONS DURING INITIAL NURSE ASSESSMENT IN THE EMERGENCY DEPARTMENT (ED)}

${ }^{1} \mathrm{C}$ Bird, 'S Hartshorn, ${ }^{2} \mathrm{~A}$ Sinclair. 'Emergency Department, Birmingham Children's Hospital, Birmingham, UK; '2Pharmacy Department, Birmingham Children's Hospital, Birmingham, UK

10.1136/archdischild-2015-308599.80

Aims Innovative ways to optimise ED patient flow, without sacrificing quality of care, are at a premium. ${ }^{2}$ Within our own paediatric ED, it was observed that inefficiency occurred whenever a triage nurse had to leave the assessment room in order to find a colleague to check the dose of a Patient Group Directive (PGD), including those for simple, over-the-counter medications. Doubt has been cast on the efficacy of double checking in all but high risk medications. ${ }^{2}$

We aimed to evaluate the safety of a "single checker" PGD process at triage for paracetamol (pain and fever), ibuprofen (pain and fever), oral rehydration salts (ORS) and topical 4\% tetracaine gel (Ametop) to improve patient flow.

Methods Single-checker PGDs were devised for the medications and indications listed above, to be used exclusively within the triage/assessment area by nurses who had completed PGD competency training. The process change was approved by the Trust Drug and Therapeutics Committee, after assurance that robust safety nets were in place (including the production of weight/ dose tables for paracetamol and ibuprofen which were displayed in the assessment room).

At launch, a 3 month audit (August-October 2011) was conducted, in which all single checker PGDs were logged.

Subsequently, the hospital incident reporting system was reviewed for any medication errors associated with PGDs from ED.

Results During the first 3 months of the use of single-checker PGDs, no errors in dose were identified.

To date, no medication errors associated with ED PGDs have been identified within the hospital incident reporting system.

Benchmarking data regarding the prevalence of this practice within EDs in the PERUKI network will be identified.

Conclusion There were no drug errors with single checking by protocol of simple emergency medications at triage, within one of the UK's busiest paediatric EDs. Further research is required to quantify the time and resources saved on the patient journey.

\section{REFERENCES}

1 Sinclair D. Emergency department overcrowding - implications for paediatric emergency medicine. Paediatrics Child Health 2007;12:491-494

2 David U. Double checking: does it work? Can J Hosp Pharm. 2003;53:167-169

\section{G82(P) EXPLORING THE ACCEPTABILITY OF A CLINICAL DECISION RULE TO IDENTIFY PAEDIATRIC BURNS DUE TO MALTREATMENT}

${ }^{1}$ EL Johnson, 'SA Maguire, ${ }^{2} \mathrm{LI}$ Hollén, ${ }^{1} \mathrm{AM}$ Kemp. 'College of Biomedical and Life Sciences, Cardiff University, Cardiff, UK; ${ }^{2}$ Centre for Child and Adolescent Health, University of Bristol, Bristol, UK

10.1136/archdischild-2015-308599.81
Objective A Clinical Decision Rule (CDR) was developed from a systematic review and epidemiological study to identify burns due to child maltreatment. Prior to an implementation evaluation, we wish to explore clinician's response to the CDR, and the likelihood that it would influence their decision making.

Methods A semi-structured questionnaire of 55 Health professionals in 8 Emergency Departments (3 paediatric) and two burns unit's explored demographics, recognition of maltreatment utilising four case vignettes (1: suspect maltreatment, 2: consider maltreatment, 1: likely unintentional), and likelihood of taking action recommend by CDR. Analysis: Fisher's exact test and logistic regression.

Results In an analysis of potential variables, (professional grade, child protection (CP) training or paediatric burns training), the most influential in accurately identifying maltreatment was professional grade (Odds Ratio 2.95, 95\% CI 1.39-6.25). Lower grade doctors were most likely to take the action recommended by the CDR, whilst higher grade doctors would do so with a proviso e.g. senior CP colleague advice. More CP training did not correlate to accuracy in identifying suspected or concerning cases, but did correlate with correctly identifying the unintentional case $(\mathrm{p}=0.041)$ and with a proviso to taking CDR recommended action $(p=0.056)$. Paediatric burns training was not an influential variable.

Conclusions While lower grade doctors are the least accurate at identifying burns due to maltreatment, they are the most likely to follow this CDR. However, those with the least knowledge of $\mathrm{CP}$ are least likely to follow the CDR recommended action.

\section{G83(P) EMERGENCY DEPARTMENT MANAGEMENT OF CHILDREN WITH DECOMPENSATING INHERITED METABOLIC DISEASE}

${ }^{1} \mathrm{MW}$ Gillam, ${ }^{2}$ E Chronopoulou, ${ }^{3} \mathrm{MD}$ Lyttle. 'General Practice, Great Western Hospital, Swindon, UK; ${ }^{2}$ Emergency Medicine, Royal Bristol Hospital for Children, Bristol, UK; ${ }^{3}$ Metabolic Medicine, Royal Bristol Hospital for Children, Bristol, UK

\subsection{6/archdischild-2015-308599.82}

Background and aims Metabolic decompensation may occur in patients with disorders of intermediary metabolism during intercurrent illness. Early intervention strategies are crucial in order to halt decline. This poses a particular challenge in emergency departments (EDs), particularly at peak times. Impending deterioration may not be clinically apparent, and so may not result in prioritisation for initial assessment or subsequent triage categorisation. It is therefore crucial to ensure appropriate care pathways are in place, yet there are no existing national guidelines regarding timeliness of ED assessment for these patients. We therefore aimed to assess current practice to inform service development. Methods Retrospective electronic database and medical chart review over a 3 month period. Nine standards were set through consensus between the ED and inherited metabolic disease (IMD) teams, relating to timeliness, notifications and assessment criteria (grade of clinician and assessment performed).

Results Of 38 IMD presentations, 30 were deemed at risk of decompensation due to their condition. 33 (92\%) had an electronic diagnosis alert, $28(83 \%)$ had a specific electronic ED management plan. 21 (54\%) were triaged within $15 \mathrm{~min}$ of arrival, and following triage $8(21 \%)$ and $19(50 \%)$ were seen within 10 and $30 \mathrm{~min}$ respectively. There was no apparent correlation with triage category. 8 were discharged prior to senior review 6 of which were at risk of decompensation. 13 of the 
30 patients at risk of decompensation received IV/NG fluids 5 of which were within an hour.

Conclusions Patients at risk of decompensation due to an inborn error of intermediary metabolism are difficult to identify, and subsequently may not fulfil criteria for urgent assessment. Use of electronic alerts, in conjunction with training, departmental policies, and tailored management plans are likely to improve care, and have been implemented. Clarity of communication is key, specifically in identifying those most likely to deteriorate unexpectedly. However, this study was not designed to identify whether or how quickly any patient deteriorated. Further multicentre work is therefore crucial to delineate optimal assessment and intervention timelines, and will result in the development of national standards that will improve both short and long term outcomes for this vulnerable group.

\section{G84(P) CULTURAL RISK OF FOREIGN BODY ASPIRATION}

${ }^{1} \mathrm{O}$ Aziz, ${ }^{2} \mathrm{~K}$ Berry, ${ }^{1} \mathrm{~N}$ Makwana. ${ }^{1}$ Paediatrics, Sandwell and West Midlands NHS Trust, West Bromwich, UK; '2Emergency Department, Birmingham Children's Hospital, Birmingham, UK

\subsection{6/archdischild-2015-308599.83}

Introduction Wearing a head scarf is practised by certain cultures and religions including Muslim females. The head scarf may be fixed using pins, there have been previous case reports and case series of accidental inhalation of scarf pins from countries where wearing head scarves is common, such as Egypt, Kuwait and Turkey. We present a case series from a city in the UK, of females with accidental scarf pin inhalation.

Methods We searched for patients who had accidental scarf pin inhalation/indigestion presenting to a tertiary care Children's Hospital emergency department and also those that were referred to the Acute Paediatric Department at a District General Hospital from January 2008 to December 2014.

Results Over a 6 year period, a total of 12 patients presented with accidental scarf pin inhalation/ingestion. The age range of these patients ranged between 11 to 14 years, with a mean of 12.3 years. All were female Muslims, with no significant past medical history. The history given was that of the scarf pin was held in the mouth, and patients reported that they sneezed, were talking at the same time, laughing or were pushed by another person as a result of which they ingested or inhaled the pin. In 9 out of 12 of these patients, the x-ray's showed that the pin was in the stomach, and 6 of these patients had a repeat $\mathrm{x}$-ray to ensure that the pin had progressed within the GI tract. In two patients the pin was inhaled and was found to be in the left main bronchus and in one patient the pin was found to be in the upper airway on chest $\mathrm{x}$-ray. All three patients required laryngotracheobronchoscopy and foreign body removal.

Conclusion Health professionals working in a multi-cultural area need to be aware of this presentation and appropriate management. Wider educational measures are required in order to increase awareness regarding avoidance of placing the scarf pins in the mouth whilst placing head scarves.

\section{G85(P) FOLLOW UP OF ASYMPTOMATIC MICROSCOPIC HAEMATURIA/PROTEINURIA - DIFFERENT PERSPECTIVES OF PAEDIATRIC EMERGENCY MEDICINE, GENERAL PAEDIATRICS AND PAEDIATRIC NEPHROLOGY}

${ }^{1}$ RA Hastings, ${ }^{2}$ J Surridge, ${ }^{3}$ AJ Lunn. ${ }^{1}$ General Paediatrics, Nottingham Children's Hospital, Nottingham, UK; ${ }^{2}$ Children's Emergency Department, Derbyshire Children's Hospital, Derby, UK; ${ }^{3}$ Children's Renal and Urology Unit, Nottingham Children's Hospital, Nottingham, UK

\subsection{6/archdischild-2015-308599.84}

Aims To examine the practice of different sub-specialists with regard to the follow up of microscopic haematuria/proteinuria as an incidental finding in febrile children.

Methods Data from an online survey of responses to hypothetical scenarios of febrile children with incidental findings of microscopic haematuria/proteinuria in children with no overt signs of renal disease was collected. The survey was sent to registrars and consultants in paediatric emergency medicine, in paediatric nephrology and in general paediatrics. It asked for the doctors' current practice in arranging follow up of children with positive urine dipstick results.

Results A response to the survey was obtained from 34 paediatric emergency medicine (PEM) specialists, 10 paediatric nephrologists (PN) and 19 general paediatricians (PG). Table 1 shows the current practice of these doctors in managing children with $1+$ positive urine dipstick $(1+\mathrm{P} / \mathrm{H}$; either protein or blood $)$ and with $3+$ proteinuria and $3+$ haematuria $(3+\mathrm{P} \& \mathrm{H})$. Amongst consultants, PEM doctors were $3 \mathrm{x}$ more likely to investigate $3+$ haematuria and proteinuria as inpatients when compared with renal consultants. These differences suggest a trend towards increased follow-up recommended by paediatric nephrologists but with fewer admissions which was not statistically significant (Pearson Chi squared test).

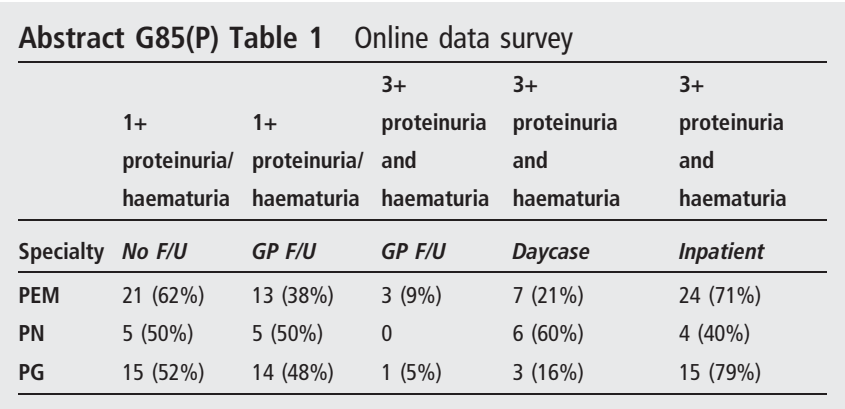

Conclusions An incidental finding of haematuria/proteinuria is common in CED. Approximately 2/3 of microscopic haematuria/ proteinuria in children without specific renal symptoms resolves. Ensuring resolution is important since up to $50 \%$ of children in whom it persists have renal disease. ${ }^{1}$ Here we show a trend for nephrologists to follow up more urine dipstick results but for PEM doctors to admit more children for inpatient investigations. PEM doctors and general paediatricians tend to have similar patterns of follow up.

\section{REFERENCE}

1 Geary DF, Schaefer F. Comprehensive Pediatric Nephrology. Philadelphia: Mosby, 2008 